\title{
MICROFLUIDIC SYSTEM SIMULATION INCLUDING THE ELECTRO-VISCOUS EFFECT
}

\author{
Eileen Rojas and C. P. Chen \\ University of Alabama in Huntsville \\ Huntsville, AL 35899
}

\author{
Alok Majumdar \\ NASAMarshall Space Flight Center \\ MSFC, AL 35812
}

\begin{abstract}
This paper describes a practical approach using a general purpose lumped-parameter computer program, GFSSP (Generalized Fluid System Simulation Program) for calculating flow distribution in a network of micro-channels including electro-viscous effects due to the existence of electrical double layer (EDL). In this study, an empirical formulation for calculating an effective viscosity of ionic solutions based on dimensional analysis is described to account for surface charge and bulk fluid conductivity, which give rise to electro-viscous effect in microfluidics network. Two dimensional slit micro flow data was used to determine the model coefficients. Geometry effect is then included through a Poiseuille number correlation in GFSSP. The bi-power model was used to calculate flow distribution of isotropically etched straight channel and $\mathrm{T}$-junction microflows involving ionic solutions. Performance of the proposed model is assessed against experimental test data.
\end{abstract}

\section{INTRODUCTION}

Microfluidics channel networks $[1,2]$ are being placed on lab-on-a-chip devices to perform multi-step biochemical processes, or to perform parallel, arrayed biochemical experiments $[1,2]$. In these microfluidic channel networks, typical length-to-width aspect ratios are very high (usually in thousands). Traditional CFD (computational fluid dynamics) methods are not practical for microfluidics-network design. System level (or lumped parameter models) tools $[3,4,5]$ were recently developed for modeling electro-osmotic pumping, diffusion, lab-on-a-chip layout and design. When considering liquid (particularly electrolytic) flows in micron-sized channels, interfacial effects due to the existence of the electrical double layer (EDL) cannot be ignored. Surface charge occurs when solid surfaces are in contact with aqueous solutions due to the asymmetric dipoles of the molecules that reside at the interface. In microfluidic networks, the EDL induced electrokinetic effects, namely, electro-osmosis and streaming potential are well known [6]. In the absence of external applied electrical potential, an important electrokinetic effect for pure pressure driven micro flows is the so-called electro-viscous retardation effect. When a pressure gradient is applied to fluid flow through a micro channel, a streaming electric field is generated due to the EDL. This streaming potential is counter directional to the fluid and causes flow retardation. This phenomenon manifests itself in an apparent viscosity, which is larger than the actual fluid viscosity according to several theoretical and experimental studies [7-11]. The purpose of this study is to develop a simplified electrokinetic model based on dimensional analysis to account for the electroviscous effect in pressure driven microfluidic network. The model is validated against experimental studies.

\section{MODELING APPROACH}

To account for the electro-viscous effect, a body force term, resulting from the existence of surface charge and the resulting $\mathrm{EDL}$, is added to the fluid momentum equations (neglecting gravity force in typical microfluidic networks), written as: 


$$
\frac{\partial \rho u_{i}}{\partial t}+\frac{\partial}{\partial x_{j}} \rho u_{j} u_{i}=-\frac{\partial p}{\partial x_{i}}+\frac{\partial \tau_{i j}}{\partial x_{j}}+\rho_{e} E_{i}
$$

The last term of the equation (1) represents the electrokinetic effect. For streaming potential induced by the applied pressure,

$$
\lambda_{0} E_{i}=-u_{i} \rho_{e}
$$

and the local electric charge density, $\rho_{e}$, is governed by the Poisson equation

$$
\nabla^{2} \psi=-\frac{\rho_{e}}{\varepsilon \varepsilon_{0}} .
$$

In the above equations, $\rho$ is the fluid density, $u_{i}$ the Cartesian velocity components, $\mathrm{p}$ the pressure, $\tau_{i j}$ the stress tensor, $\varepsilon$ the fluid permittivity, and $\lambda_{0}$ the bulk fluid conductivity. The charge density is expressed as $\rho_{e}=e\left(Z_{+} n_{+}+Z_{-} n_{-}\right)$and the ionic number concentration, $n_{ \pm}$, can be obtained by solving the Nernst-Planck equation. Under equilibrium situation, the ion distribution can be described by the exponential function; equation (3) becomes the well-known Poisson-Boltzmann equation:

$$
\nabla^{2} \psi=\frac{2 Z e n_{0}}{\varepsilon \varepsilon_{0}} \sinh \left(\frac{Z e \psi}{k_{B} T}\right)
$$

The characteristic EDL thickness can be defined based on the inverse of the Debye-Huckel parameter $\kappa$, and $\kappa^{2}=\left(2 Z^{2} e^{2} n_{0}\right) /\left(\varepsilon \varepsilon_{0} k_{B} T\right)$. Assuming Newtonian fluids, the net effect of the EDL (last term of equation (1)) would produce extra resistance force to the fluid flow. Previous theoretical studies strive to derive analytical form of an apparent or augmented viscosity. Most assumptions involved fully developed flow field, constant fluid properties, and most importantly, the Poisson-Boltzmann equation (either the linear or non-linear form) for EDL. With the Boltzmann assumption, in which the ionic concentration follows an exponential distribution, the analytical forms of apparent viscosity usually have explicit Debye-Huckel parameter $(\kappa)$ dependency (see [7-10]). Since $\kappa$ is proportional to the square root of ionic molar concentration, formulations based on the Boltzmann assumption would lead to singularity for very diluted electrolyte solutions (see the discussion in [10] and chapter 3 of [7]). To avoid this anomaly, the Nernst equation was used to obtain the ionic concentration distribution [12] and a closeform formulation cannot be obtained.

In this study, a different approach was taken. We proposed an empirical form of the apparent viscosity formulation using surface charge and fluid's bulk conductivity as two extra dependent variables to account for the electro-viscous effect based on dimensional analysis [13]. The two dimensionless groups based on the above-mentioned parameters are:

$$
\Pi_{1}=\frac{L^{2} \lambda_{0}}{\varepsilon \nu}, \quad \Pi_{2}=\frac{\varepsilon^{\frac{1}{2}} \zeta_{0}}{L \Delta P^{\frac{1}{2}}}
$$

where $L$ is the length of the channel, $\Delta P$ is the pressure difference, $\zeta_{0}$ is the zeta potential, and $v$ is the fluid kinematic viscosity. A bi-power law form was used such that

$$
\frac{\mu_{e f f}}{\mu}=1+\mathrm{a}\left(\Pi_{1}\right)^{\mathrm{b}}\left(\Pi_{2}\right)^{\mathrm{c}}
$$

To determine the model coefficient, the slit microflow experiments of Ren et al. [11] were used to determine the constants $\mathrm{a}, \mathrm{b}$, and $\mathrm{c}$ in the functional relationship for the effective viscosity, yielding the correlation:

$$
\frac{\mu_{e f f}}{\mu}=1+0.856\left(\Pi_{1}\right)^{1.10}\left(\Pi_{2}\right)^{1.72}
$$

The slit flow of [11] was assumed to be two dimensional. To account for various channel cross section geometries encountered in microfluidic networks, the approach utilized in GFSSP (Generalized Fluid System Simulation Program, [14]) was used through the Poiseuille number correlation. This procedure consists of the following steps:

1. Estimate the hydraulic diameter of the cross-section:

$$
\mathrm{D}_{\mathrm{h}}=(4)(\text { Area }) / \text { Perimeter }
$$

2. Estimate the Poiseuille number for a particular cross-section. The Poiseuille number can be expressed as a polynomial function of aspect ratio. Coefficients for different geometries can be found in [14].

$$
P o=A_{0}+A_{1}\left(\frac{b}{a}\right)+A_{2}\left(\frac{b}{a}\right)^{2}+A_{3}\left(\frac{b}{a}\right)^{3}+A_{4}\left(\frac{b}{a}\right)^{4}
$$

3. Calculate the friction factor for a non-circular pipe:

$$
f=\frac{4 P o}{\operatorname{Re}}, \quad \text { for Laminar flow }(\operatorname{Re}<2300)
$$

GFSSP employs a finite volume formulation of mass and momentum conservation equations in a network consisting of nodes and branches. Mass conservation equation is solved for pressures at the nodes while the momentum conservation equation is solved at the branches to calculate flowrate. For given pressure drop across various ports of the microfluidic network, the GFSSP program calculates fluid velocities in each branch and pressure at each node. Further detailed numerical method utilized in the GFSSP program can be found in Majumdar et al. [14].

\section{EXPERIMENTAL PROCEDURE}

Microfluidics experimental study was carried out on a quartz chip using the Application Development Unit (ADU). 
The quartz chip consists of two joined layers. The bottom layer contains seven isotropically etched channels with an identical height and width of 15 and $50 \mu \mathrm{m}$, respectively. The top layer has 25 wells that align with the endpoints of the microchannels. The resulting chip thickness is $1.462 \mathrm{~mm}$ and the isotropically etched channel cross-section can best be described as bowlshaped. Figure 1 shows the various microchannel configurations. In this study, only the straight channel (label number 4) experiment was performed. Housed by the ADU, the chip is placed on a frame-like platform which resides above three, face-up, movable microscope lenses. A head gasket directly above the platform provides a seal when lowered onto the chip. Specific pressures can then be applied to any of the 25 wells. The ADU has a pressure range of -5 to 5 psi. The setup is shown in Figure 2.

A data acquisition and GUI control system (LabView) is used to manage the ADU. Well pressure, lens magnification, focus and movement, and light intensity can be adjusted by the control system. The magnified microchannel image is displayed on an adjacent monitor, and fluid movement can be observed during operation. Hash marks of known length along certain channels were used to indicate start and stop times for subsequent velocity calculations.

Two different concentrations of aqueous $\mathrm{KCl}$ solutions were used as testing liquids: $10^{-2}$ and $10^{-4} \mathrm{M} \mathrm{KCL}$. The addition of dye was necessary for visualization of fluid movement, particularly the interface, along the channel. Aliquots of testing liquid were added using a $20 \mu \mathrm{L}$ pipette to the wells at either end of the channel to minimize capillary forces. The chip was then placed in the ADU and specific pressures were applied to one well to initiate fluid movement to the lower pressure well. To create the interface, fluid at the higher pressure well contained the dye while the fluid at the lower pressure did not. A stop watch was used to measure the interface travel time for a predetermined distance. Additional travel distance was allowed for the fluid to reach steady state. After passing the end marker, the direction of fluid flow was reversed by lowering the pressure on the well.

Pressure readings from the ADU were used to determine the pressure differential. Multiple runs at the same pressure were carried out on the same channel. The channel was cleaned with $1 \mathrm{M} \mathrm{NaOH}$ and then DI water before changing the testing liquid. The process was repeated for a range of pressures for each of the testing liquids. Only the straight channel results are presented in this paper.

\section{RESULTS \& DISCUSSION}

The proposed model (Eq.7) was implemented into the system level computer program GFSSP for predicting the straight microchannel flow (\#4 in figure 1). Table 1 shows the predicted results versus the experimental data. As seen from the table, the reduction of flow velocity due to electro-viscous effect can be observed experimentally. The degree of flow retardation is quite small for the pressure drop range used in this experiment. The model predictions also show flow reduction as expected. However, the degree of flow retardation was overpredicted. The data indicated about 10 percent reduction in flow velocity (as indicated by lower Reynolds number) from $10^{-2} \mathrm{M}$ to $10^{-4} \mathrm{M} \mathrm{KCl}$ solution flows. The model results show larger, up to 20 percent, reduction from no-EDLeffect to EDL-effect $\left(10^{-4} \mathrm{M} \mathrm{KCl}\right)$ flows. In this study, the flowfield generated by $10^{-2} \mathrm{M} \mathrm{KCl}$ solution was assumed to be unaffected by electro-viscous effect following the suggestion of Ren et al [11]. Due to the difference in channel shape, the $10^{-2}$ $\mathrm{M} \mathrm{KCl}$ flow may exhibit electro-viscous effect in this study. This, however, was not modeled here. For the $10^{-2} \mathrm{M} \mathrm{KCl}$ case, laminar simulation results (without EDL effect) were used for comparison. The pressure drops used in this study were also much lower than those used in the slit-flow experiment of Ren et al. [11]. At higher pressures drops, the current flow visualization technique used for flow velocity measurement becomes unreliable. The model did show consistent trend in this straight channel case.

Next, we turned to the application of the current model to the T-junction micro flow on the Caliper N145 chip [15]. Figure 3 shows the layout and dimensions of micro-fluidic chip considered in the present study. The glass chip was micro fabricated by isotropic etching with about 60 micrometer in width and 12.4 micrometer in depth. The corresponding GFSSP model is shown in Figure 4. By adjusting the pressure difference among well number 1, 8 and 4 , different flow fields across the T-junction can be set up. Dyes were introduced in well number 1 and well number 8 for flow velocity measurement [5] using vitalization technique. To accommodate flow visualization, pressure levels at well number 1,8 and 4 were always different with well number 4 being the minimum. For micro-channel with this dimension, the electro-viscous effect would be significant for water and low concentration ionic solution fluid flows. In addition to pure water, aqueous $\mathrm{KCl}$ solutions of concentration $10^{-4}$ and $10^{-2} \mathrm{M}$ were used as the testing liquids. Following the previous studies [7, 11], the $10^{-2} \mathrm{M}$ experiment was taken to be the case without EDL effects when compared to the numerical results. Figure 5 shows the comparison between numerical prediction and test data. The velocity in branch 34 is plotted with the maximum pressure differential between inlet and outlet. Experimental data has been compared with GFSSP prediction. The variation between the $10^{-2}$ and $10^{-4} \mathrm{M} \mathrm{KCl}$ velocities increases as the pressure difference increases. At higher $\triangle \mathrm{P}$ values, conventional laminar result overestimates the velocity of the $10^{-4} \mathrm{M} \mathrm{KCl}$ solution, which is considered to be affected by the EDL. When the electro-viscous effect is taken into consideration, the results show good comparison against experimental data for $10^{-4} \mathrm{M}$ $\mathrm{KCl}$ with no more than a $5 \%$ difference. The electroviscous effect accounts for a decrease in velocity of up to $17 \%$ for the experimental data. Therefore, while the $10^{-2} \mathrm{M} \mathrm{KCl}$ solution was not affected by the EDL, the $10^{-4} \mathrm{M} \mathrm{KCl}$ solution required GFSSP to consider the electroviscous effect for better predictions.

\section{CONCLUSIONS}

An empirical model suitable for system-level lumped parameter analysis was proposed to account for electro-viscous effects in microfluidic network. The electro-viscous effects arise from the electrical double layer (EDL) naturally formed 
between flowing ionic solutions and solid walls. Based on dimensional analysis, the current model includes surface charge and fluid bulk conductivity as two extra parameters, in addition to conventional parameters used in pressure-driven laminar flows. The proposed bi-power form was used to calculate an augmented viscosity and a Poiseuille number correlation was used for channel geometry effect. The model was tested against experimental data of flow distribution in isotropically etched straight channel flow and $\mathrm{T}$-junction microflow involving ionic solutions. For the straight channel microflow, the flow rate retardation of $10 \%$ was observed experimentally. The model, however, overpredicts the retardation in straight channel flow. Explanation of this discrepancy was discussed. In T-junction microflow, as much as $17 \%$ flow retardation was observed in high pressure drop case, and the model predictions compare favorably for this case. Further improvement on flow velocity measurement is required. Testing of the model in more complicated networks and extension of the model to include entrance effect are the subjects of on-going studies.

\section{ACKNOWLEDGMENTS}

The authors wish to acknowledge the Lab-On-a-Chip Application Development (LOCAD) lab of NASA-Marshall Space Flight Center for providing the ADU platform for microfluidics testing, as well as the support from the National Science Foundation Bridge to the Doctorate Fellowship Program.

\section{REFERENCES}

1. Oosterbroek, R. E. and van den Berg, A., Eds., 2003, Labon-a-chip: miniaturized systems for (bio) chemical analysis and synthesis, Elsevier, the Netherlands.

2. Smith, L. A. and Sobek, D., Eds, 2004, Lab-on-a-chip: platforms, devices, and applications, Proceedings of SPIE, Vol. 5591.

3. Qiao, R. and Aluru, N. R., 2002, "A compact model for electroosmotic flows in microfluidics devices," J. Micromech. Microeng., Vol. 12, pp. 625-635.
4. Bedekar A. S. et al., 2006, "System-Level simulation of flow-induced dispersion in Lab-on-a-Chip systems," IEEE Tran. Computer-Aided Design of Integrated Circuits and Systems, Vol. 25, pp. 294-304.

5. Ajdari, A., 2005, "Steady flows in networks of microfluidics channels: Building on the analogy with electrical circuit," Comptes Rendus Physique, Vol. 5, pp. 539-546.

6. Butt, H.-J., Graf, K. and Kappl, M., Physics and Chemistry of Interface, 2003, Wiley-VCH.

7. Li, D., Electrokinetics in Microfluidics, 2004, Elsevier.

8. Vainstein, P. and Gutfinger, C., 2002, "On electroviscous Effects in Microchannels," J. Micromech. Microeng., vol. 12, pp. 252-256.

9. Chun, M.-S., Lee, T. S. and Choi, N. W., "Microfluidic analysis of electrokinetic streaming potential induced by microflows of monovalent Electrolyte solution," 2005, J. Micromech. Microeng., vol. 15, pp. 710-719.

10. Jain, A. and Jensen, M. J., "Analytical modeling of electrokinetic effect on thermal Transport in Electrolytic flow in microchannels," 2005 , in $21^{\text {st }}$ IEEE SEMI-THERM symposium.

11. Ren, L., Qu, W. and $\mathrm{Li}$, D., 2001, "Interfacial electrokinetic effects on liquid flow in microchannels," Int. J. Heat and Mass Transfer, vol. 44, pp. 3125-3134.

12. Ren, C. L. and Li, D., "Improved understanding of the effect of electrical double layer on pressure-driven flow in Microchannels," 2005, Anal. Chim. Acta, vol. 531, pp. 1523.

13. Rojas, E., "Microfluidics system simulation including the electroviscous effect," 2006, M. S. Thesis, The University of Alabama in Huntsville.

14. Majumdar, A., Cole, H. and Chen, C. P., 2005, "Numerical Modeling of Flow Distribution in Micro-Fluidics Systems," in Forum on Microfluidic Devices and Systems, ASME Fluids Engineering Summer Meeting, Houston, June.

15. Hunterhower, H., 2004, "Study to Aid Computer Modeling of Lab-on-Chip Technologies," Presentation at NASAMSFC, USRP Summer Program, July. 


\begin{tabular}{|c|c|c|c|c|}
\hline & $\mathrm{KCl} 10^{-2} \mathrm{M}$ & No EDL & KCl $10^{-4} \mathrm{M}$ & With EDL model \\
\hline $\begin{array}{c}\mathbf{d P} / \mathbf{d x} \\
\left(10^{6} \mathrm{~Pa} / \mathrm{m}\right)\end{array}$ & $\mathbf{R e}$, exp. & $\mathbf{R e}$, pred & $\mathbf{R e}$, exp. & Re, pred \\
\hline 0.0420 & $0.0199+/-0.00111$ & 0.0202 & & 0.0158 \\
\hline 0.0422 & & 0.0203 & $0.0187+/-0.001185$ & 0.0159 \\
\hline 0.0794 & & 0.0384 & $0.0363+/-0.003$ & 0.030 \\
\hline 0.0810 & $0.0364+/-0.00207$ & 0.0390 & & 0.0305 \\
\hline
\end{tabular}

Table 1. Comparison between the model prediction and experimental data

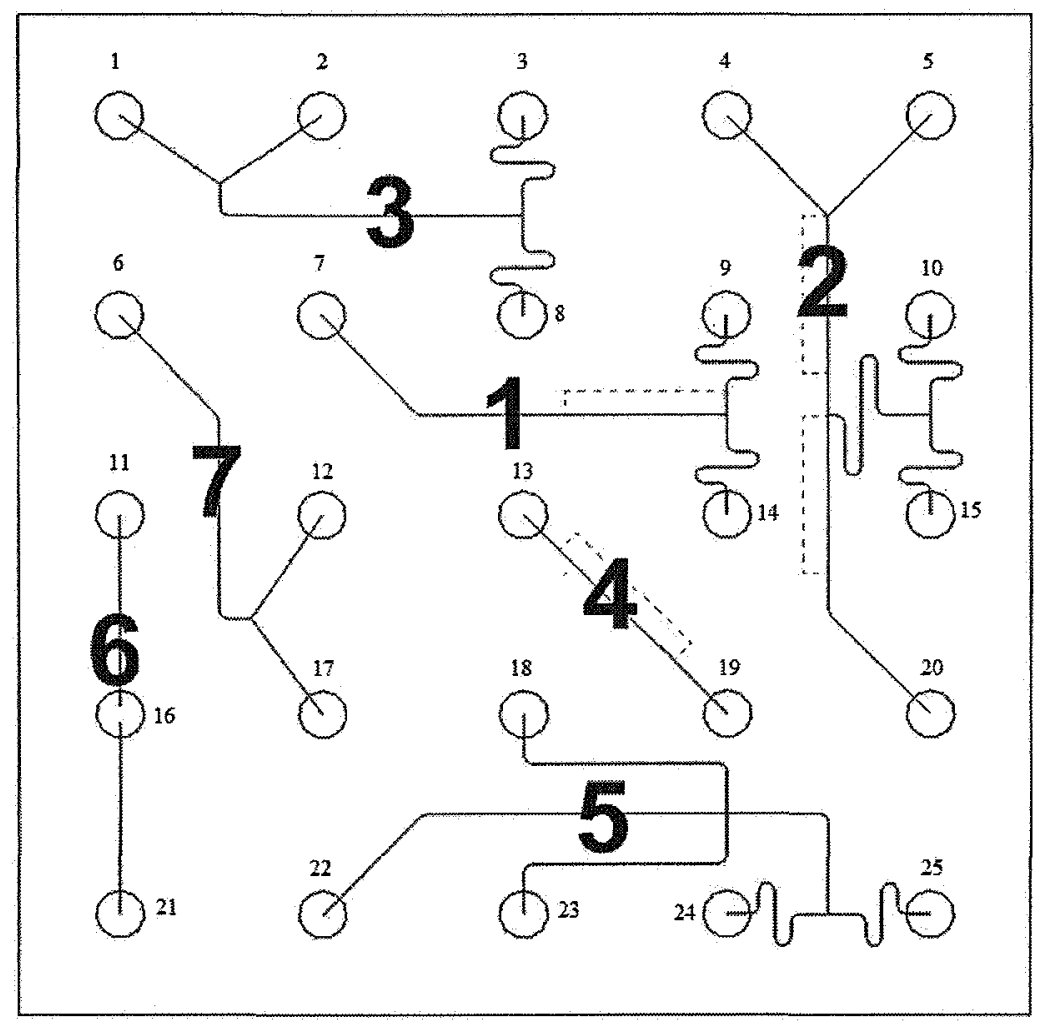

Figure 1. Layout of microfluidic chip 


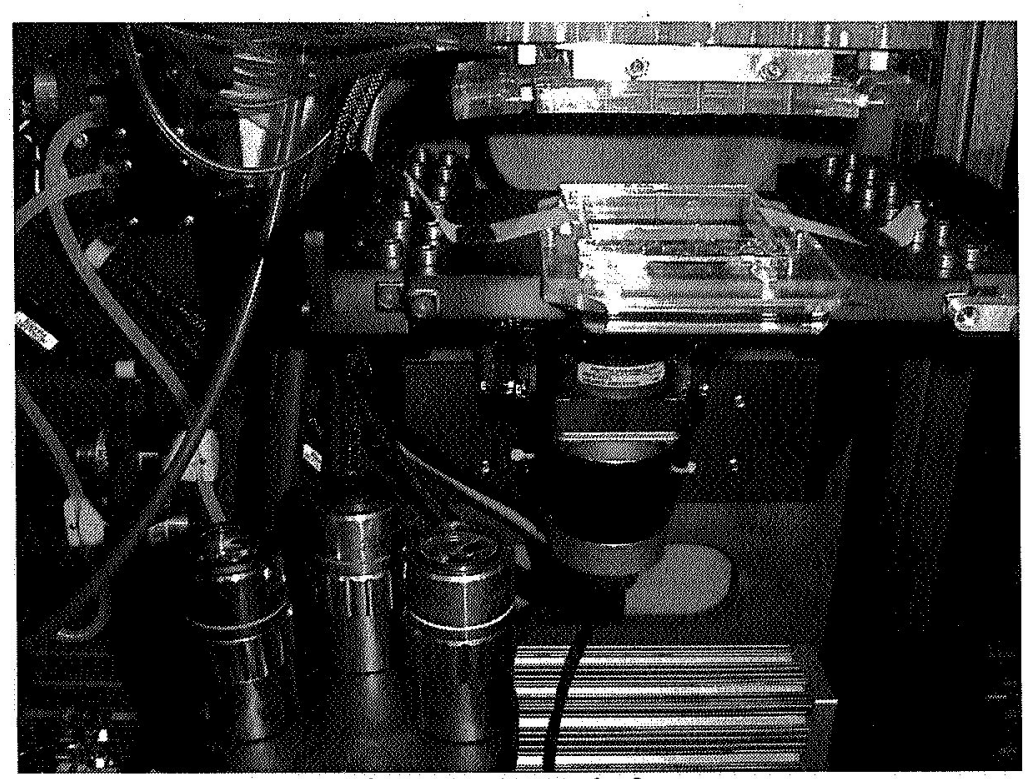

Figure 2. ADU platform

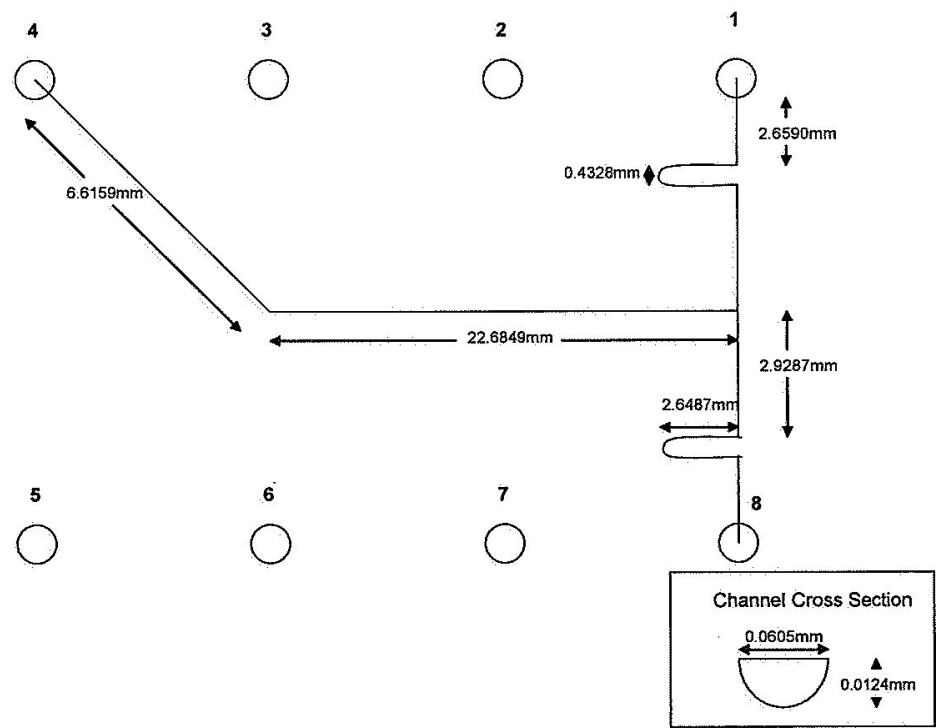

Figure 3. Micro-fluidic chip considered for model verification 


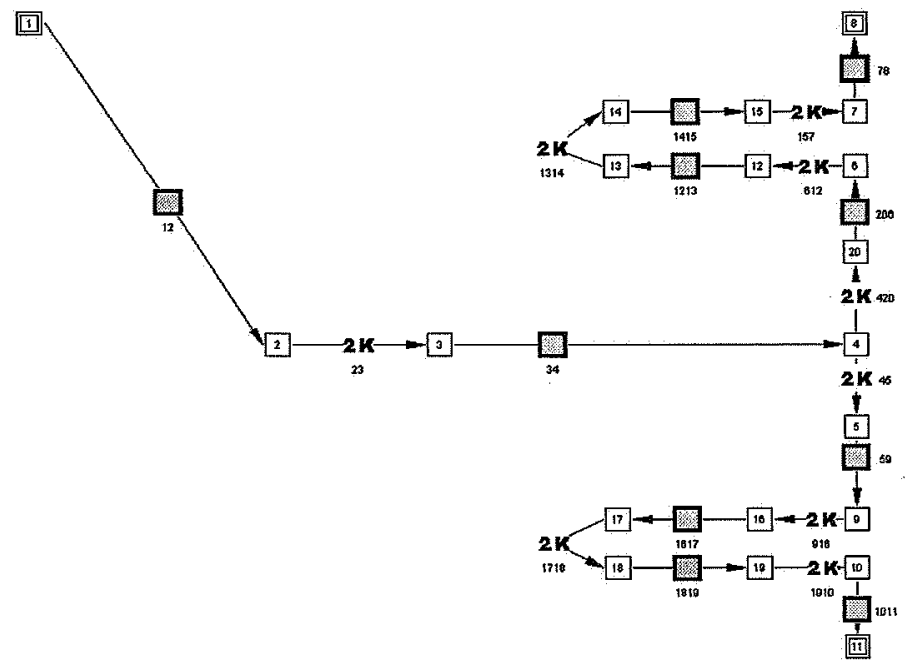

Figure 4. GFSSP model of flow network in chip with micro-channels

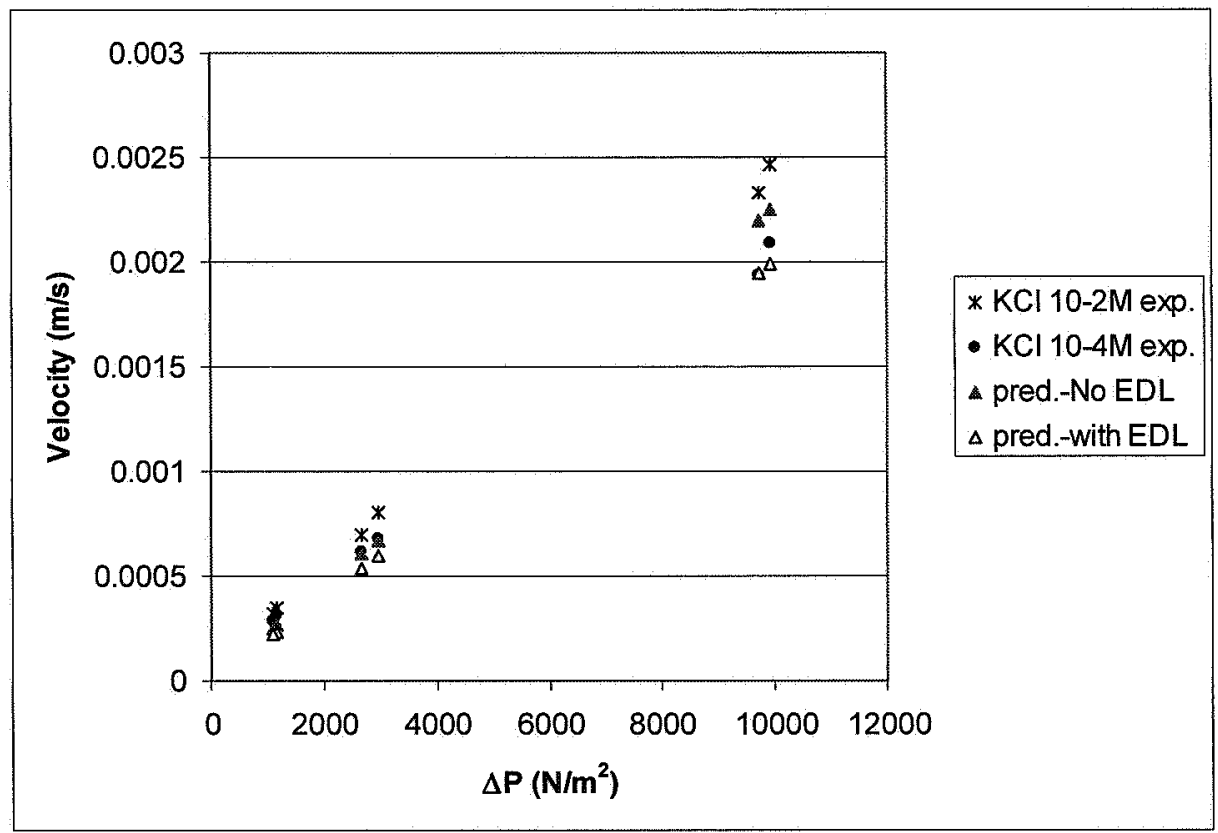

Figure 5. Comparison between current model predictions and test data 